Meeting report

\title{
La cellule vue par l'Etablissement français des Greffes : aspects réglementaires
}

\author{
D. Houssin et G. Novakovitch ${ }^{1}$
}

Etablissement Français de Greffes, ${ }^{1}$ Département Médical et Scientifique, 28, rue de Charenton, F-75571 Paris Cedex 12, France

L'activité thérapeutique intégrant un support cellulaire comporte, outre l'indication de greffe de cellules et tous les aspects cliniques liés à cette activité, une série d'actes dont l'ensemble doit répondre à des critères de qualité.

Dans l'allogreffe de cellules souches hématopoïétiques de moelle osseuse, de sang périphérique et de placenta, l'appariement immunologique, donc la qualité des examens de laboratoire nécessaires à cette détermination, est capitale. La promotion de la qualité de cet appariement est une des missions de l'Etablissement français des Greffes (I'EfG) décrétée en Octobre 1994.

Les mesures permettant d'assurer la meilleure sécurité possible dans ce domaine sont proposées par l'EfG et, notamment, en ce qui concerne tous les produits entrant en contact avec les cellules d'origine humaine du prélèvement à la greffe.

En collaboration, l'EfG et l'AFS souhaitent que des fiches de spécification des produits d'origine chimique ou biologique entrant en contact avec les cellules, soient établies et contrôlées par l'Agence du Médicament. Le prélèvement de cellules, la transformation du produit cellulaire y compris son éventuelle conservation et le contrôle de qualité de celui-ci au moment de la greffe ou de l'injection devront être pratiqués dans le cadre de bonnes pratiques dont la rédaction incombe à l'EfG.

Dans sa mission de coordination de greffes de cellules, l'EfG conseille la Direction Générale de la Santé et la Direction des Hôpitaux notamment, dans l'élaboration des textes concernant l'autorisation délivrée à des établissements pour pratiquer l'activité de greffe de cellules souches hématopoïétiques.

Enfin, le recueil des informations nécessaires à l'évaluation des activités de prélèvement et de greffe permet à l'EfG d'assumer sa mission d'évaluation et d'analyse des résultats obtenus par type de greffe et par équipe.

L'écriture des règles de bonnes pratiques relatives au prélèvement, à la transformation y compris la conservation, au transport, au contrôle de la qualité des produits cellulaires, que les cellules soient destinées à être modifiées ou pas, est terminée.

Ce texte, qui a fait l'objet d'une large concertation, a été approuvé par les trois établissements publics concernés, l'Agence Française du Sang (I'AFS), I'Agence du Médicament (I'AdM) et l'Etablissement français des Greffes.

Le prélèvement, la transformation des produits cellulaires, leur contrôle de qualité se dérouleront dans des sites autorisés par le Ministre chargé de la santé après avis de l'EfG et de l'AFS selon que ces actes sont réalisés dans un établissement de transfusion sanguine ou un établissement de santé.

L'EfG et l'AdM collaborent afin qu'un contrôle de qualité particulier des laboratoires d'histocompatibilité pratiquant des examens en vue de greffe, soit instauré.

Une réflexion avec les directions du ministère chargé de la santé est menée à propos des greffes de cellules et concerne l'organisation en réseau de cette activité de soins.

Cette réflexion sera enrichie par l'élaboration des règles de bonnes pratiques cliniques de greffes et injections de cellules d'origine humaine dont l'écriture débute ce mois d'octobre.

En conclusion, des chantiers ont été ouverts, certains le seront très prochainement alors que d'autres le resteront tant ce domaine est en pleine évolution et transformation. 
Back to the HCT-EE Home Page

Last change: April 28, 1997

helpdesk.link@springer.de

() 1996 by Springer-Verlag France 